OPEN

SUBJECT AREAS:

ENDOCYTOSIS

SMALL GTPASES

Received

31 October 2013

Accepted

13 November 2014

Published

2 December 2014

Correspondence and requests for materials should be addressed to

W.J.H. (mcbhwi@ imcb.a-star.edu.sg) or

T.L.W. (xmuibrwt!@ xmu.edu.cn)

* These authors contributed equally to this work.

\section{RILP interacts with HOPS complex via VPS4 1 subunit to regulate endocytic trafficking}

Xiaosi Lin '*, Ting Yang ' *, Shicong Wang' 'Zhen Wang' ', Ye Yun' ', Lixiang Sun', Yunhe Zhou' ', Xiaohui Xu', Chihiro Akazawa², Wanjin Hong ${ }^{1,3}$ \& Tuanlao Wang'

${ }^{1}$ School of Pharmaceutical Sciences, State Key Laboratory of Cellular Stress Biology, Xiamen University, Xiamen, Fujian, China, $361005,{ }^{2}$ Department of Biophysics and Biochemistry, Graduate School of Health Sciences, Tokyo Medical and Dental University, Yushima 1-5-45, Bunkyo-Ku, Tokyo 1 13-8519, Japan, ${ }^{3}$ Institute of Molecular and Cell Biology, A STAR (Agency for Science, Technology and Research), 61 Biopolis Drive, Singapore 138673, Singapore.

The HOPS complex serves as a tethering complex with GEF activity for Ypt7p in yeast to regulate late endosomal membrane maturation. While the role of HOPS complex is well established in yeast cells, its functional and mechanistic aspects in mammalian cells are less well defined. In this study, we report that RILP, a downstream effector of Rab7, interacts with HOPS complex and recruits HOPS subunits to the late endosomal compartment. Structurally, the amino-terminal portion of RILP interacts with HOPS complex. Unexpectedly, this interaction is independent of Rab7. VPS41 subunit of HOPS complex was defined to be the major partner for interacting with RILP. The carboxyl-terminal region of VPS41 was mapped to be responsible for the interaction. Functionally, either depletion of VPS41 by shRNA or overexpression of VPS41 C-terminal half retarded EGF-induced degradation of EGFR. These results suggest that interaction of RILP with HOPS complex via VPS41 plays a role in endocytic trafficking of EGFR.

T

he late endolysosomal trafficking in the yeast is governed by small GTPase Ypt7p/Rab7 and its GEF HOPS (homotypic fusion and protein sorting) complex ${ }^{1-4}$. Rab7 and HOPS are structurally conserved in mammalian cells. However, mammalian cells also contain a downstream effector of Rab7 called RILP (Rab interacting lysosomal protein) that is not structurally present in the yeast ${ }^{5,6}$. HOPS complex consists of 6 subunits of VPS (vacuole protein sorting) proteins, namely VPS11, VPS16, VPS18, VPS33, VPS39 and VPS41, with the former 4 subunits also referred to as class C VPS proteins. It is well established the Class C VPS proteins interact with one another, assembling into VPS-core complex, while accessory proteins, VPS39 and VPS41, associate with VPS-Core to form the complete HOPS complex ${ }^{7-10}$.

Previous studies demonstrated that HOPS complex plays a critical role in regulating the late stage of endocytic pathway, since mutations in HOPS subunits result in severe traffic disorder in yeast ${ }^{11,12}$. HOPS complex may serve as a tethering factor or putative GEF (guanine nucleotide exchange factor)for Rab7/Ypt7p to activate Ypt7p to drive late endosomal membrane tethering and fusion ${ }^{8,13}$. Recent studies uncovered that Mon1-Ccz1 complex can inactivate the activity of Rab5 and activate Rab7's activity by regulating the GEF activity of HOPS complex, indicating HOPS complex is involved in regulating early-to-late endosomal membrane transition ${ }^{14,15}$.

Despite the structural conservation of all 6 subunits of HOPS complex in mammalian cells, the functional and mechanistic aspects of HOPS complex remain less defined. The importance of the HOPS complex is underscored by the discoveries that dysfunction in HOPS complex is associated with animal diseases. Defects in VPS11, VPS16, VPS18 and VPS39 may result in aberrant pigmentation ${ }^{16-19}$. Mutation in VPS33a gene results in abnormal melanosomes and Purkinje cell loss in $b f$ mouse ${ }^{20,21}$. Furthermore, mutation in VPS33b is associated with human disease arthrogryposis-renal dysfunction-cholestasis (ARC) syndrome ${ }^{22}$. Functionally, overexpression of VPS39/ Vam6 induces clustering of enlarged late endosome/lysosomes, which is independent of Rab7's activity ${ }^{23}$. Although Vps39p may activate Ypt7p binding to GTP in yeast, no subunit of HOPS complex has been shown to directly possess GEF activity to Rab7 in mammalian cells ${ }^{13}$. The most recent study shows that HOPS can interact with clathrin and ERM (Ezrin/Radixin/Moesin) proteins to regulate endocytosis ${ }^{24}$.

RILP is a downstream effector shared by Rab7 ${ }^{5}$, Rab34 and Rab36 ${ }^{25,26}$. Despite extensive efforts, no structural counterpart of RILP is present in yeast, indicating it was evolved to accommodate the unique complexity of mammalian endocytic traffic. We have shown that dimerized RILP (through its C-terminal region) interacts 
simultaneously with two Rab7 molecules, thus recruiting/stablizing Rab7 onto the endosomal/lysosomal membrane ${ }^{25,27}$. The N-terminal region of RILP may bind to dynein/dynactin complex to drive vesicle trafficking ${ }^{6,28}$.

Both HOPS complex and RILP are crucial regulators and/or effectors for Rab7 regulating late endocytic pathway in mammalian cells. However, whether the mammalian specific RILP also engages HOPS complex in endocytic trafficking is not known. In this study, we demonstrate that the N-terminal region of RILP also interacts with HOPS complex, primarily through interaction with the C-terminal region of VPS41 subunit. This interaction is likely independent of Rab7. Furthermore, RILP-mediated membrane recruitment of HOPS subunits is compromised when VPS41 was depleted, suggesting that the interaction of VPS41 with RILP is a key event for RILP to regulate membrane recruitment of the complex. Functionally, knockdown of VPS41 retarded degradation of EGFR in response to EGF. Similarly, overexpression of C-terminal region of VPS41, which is expected to act as a dominant negative mutant via competing with endogenous VPS41 for ineraction with RILP, also retarded EGFR degradation. One possible working model is that RILP is a late endosomal integrator coordinating the activity of Rab7 and HOPS complex in addition to its interaction with other proteins such as dynein-dynactin complex and ESCRT-II subunits.

\section{Results}

HOPS subunits associate with RILP at endosomal membrane. Previous studies ${ }^{8,29}$ demonstrated that Rab7 interacts with VPS39 and VPS41 subunits of HOPS complex. In our immunofluorescence microscopy experiments, we found that epitopetagged VPS11, VPS16, VPS33 and VPS41, when expressed alone, displayed cytosolic distribution; while tagged VPS18 and VPS39, expressed alone, is spotty and vesicular. Co-expressing of HOPS subunit with Rab7 indicates that Rab7 doesn't effectively recruit HOPS to vesicles, the distribution of these 6 subunits of HOPS complex remains the same as when expressed alone (supplemental Figure s1). RILP is a downstream effector of Rab7 that is present selectively in mammalian cells. RILP can efficiently induce enlarged late endosome/lysosome as well as clustering them at the peri-Golgi region when it is overexpressed ${ }^{5,25}$. This property is mediated by interaction with Rab7, as its mutant defective in interaction with Rab7 is no longer able to induce lysosomal clustering ${ }^{27}$. Due to the observation that Rab7 does not possess the ability to recruit HOPS subunits to the endosomal membrane, we then investigated whether RILP is able to execute such an effect by co-expressing Myc-tagged HOPS subunits with GFP-RILP. As shown in Figure 1, when coexpressed, GFP-RILP was able to cause efficient recruitment of Myc-VPS11, VPS16, VPS18, VPS39 and VPS41 onto the membrane structures marked by RILP. This is to our surprise as RILP can robustly recruit most HOPS subunits (except for VPS33) to the enlarged vesicular membrane (Figure 1), in about $60-70 \%$ of cells co-expressing RILP and the VPS subunits (except for VPS33). Further examination by triple labeling of RILP, VPSs and endolysosomal marker CD63 demonstrated that RILP can recruit HOPS subunits to endolysosomal structures (Figure s2), suggesting that RILP is a key regulator for endosomal membrane recruitment of HOPS complex.

Interestingly, the enlarged/clustered structures due to RILP overexpression exhibited some different patterns depending on the coexpressed HOPS subunits. The enlarged/clustered endolysosomes due to RILP overexpression was not significantly altered when VPS16, VPS18, VPS33, or VPS41 was co-expressed (Figure 1). However, its co-expression with VPS11 or VPS39 caused more dispersed vesicular structures marked by both RILP and the VPS protein, indicating that VPS11 and VPS39 may influence the behavior of RILP but the molecular basis is not clear at the moment.

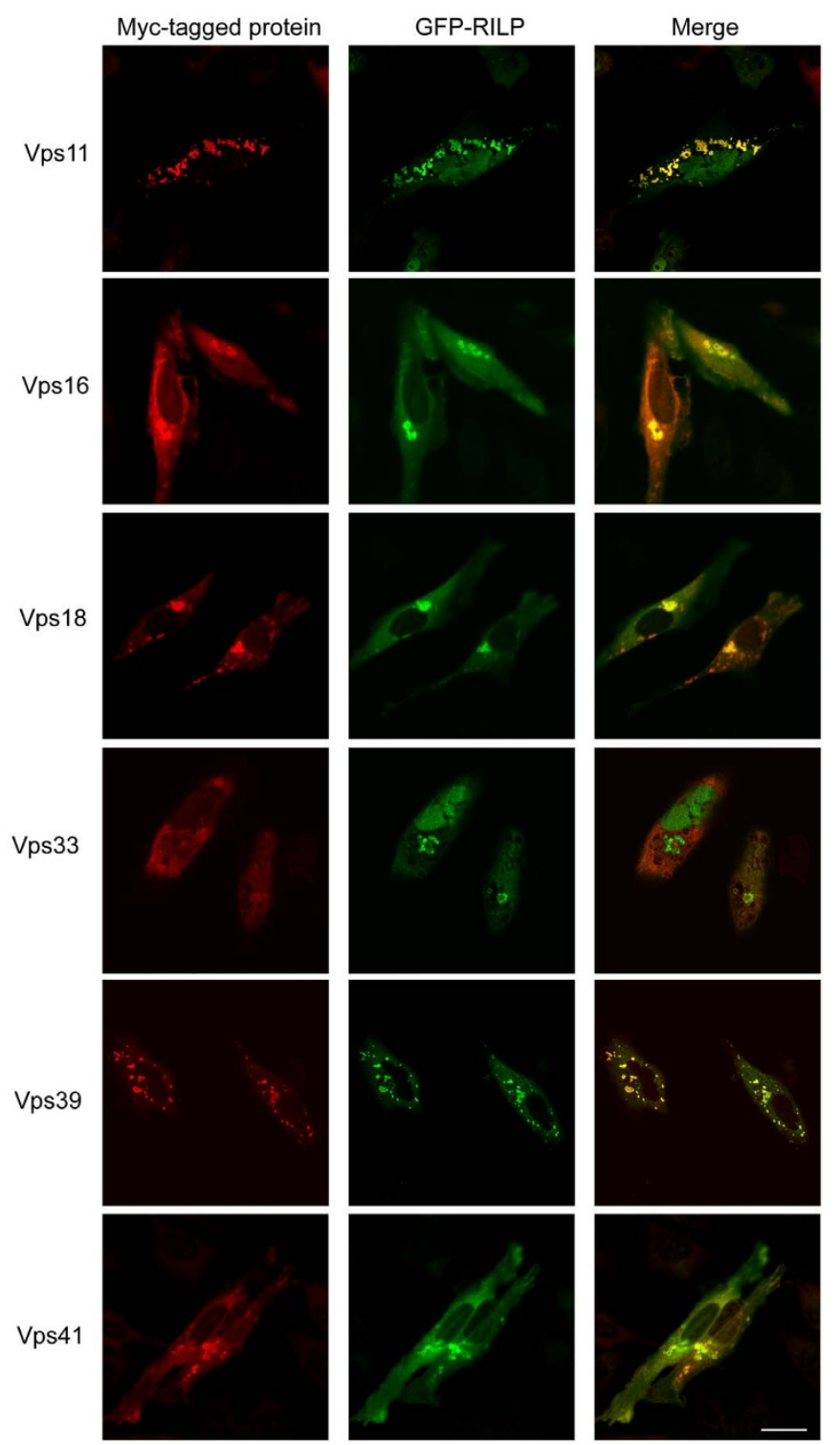

Figure $1 \mid$ RILP recruits HOPS subunits to endosomal vesicles. Hela cells were co-transfected with GFP-RILP and myc-tagged human VPS11, VPS16, VPS18, VPS33, VPS39 and VPS41, respectively. VPS proteins are immuno-labeled by 9E10 antibody followed by Texas-red conjugated secondary antibody and viewed along with GFP signal.

Immunofuorescence microscopy revealed that RILP recruits all HOPS subunits (except for VPS33) to endosomal vesicles. Bar $=20 \mu \mathrm{m}$.

HOPS subunits except for VPS33 bind to RILP. The robust membrane recruitment of 5 out of 6 HOPS subunits indicates there exists interaction between RILP with some subunits of the HOPS complex. We then investigated this via GST-pull down assay. Hela cells were transfected to express each of the Myctagged HOPS subunits and the resulting cell lysates were respectively incubated with immobilized GST-RILP. The bound proteins, along with $10 \%$ lysate, were resolved by SDS-PAGE and then analyzed by immunoblot (Figure 2A). The results revealed that the 5 subunits being recruited by RILP were efficiently retained by GST-RILP, whereas Myc-VPS33 was not retained by GST-RILP. This correlation between the in vitro interaction and in vivo membrane recruitment indicates the interaction with RILP is responsible for membrane recruitment.

Since RILP interacts with Rab7 through its Carboxyl-terminal region $^{25,27}$, the observed interaction and membrane recruitment 
A

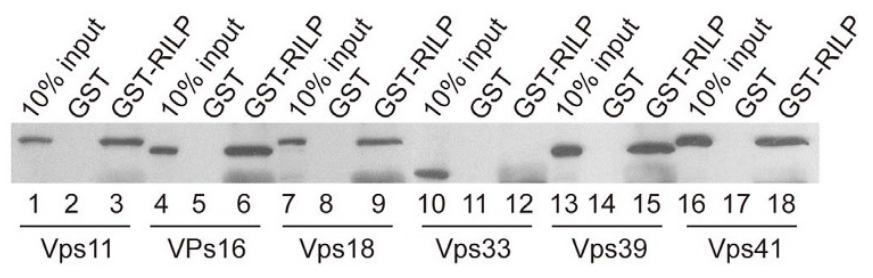

B

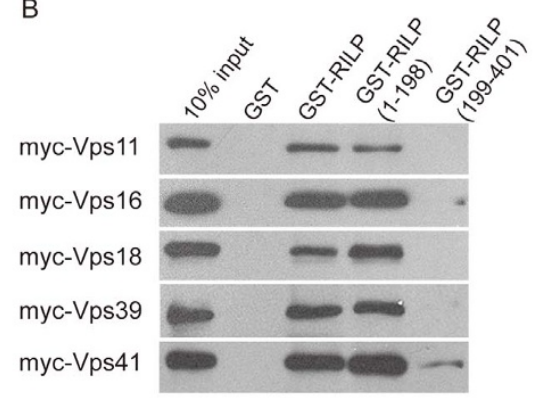

Figure $2 \mid$ RILP interacts with HOPS complex. (A). Hela cell lysates derived from cells expressing myc-tagged HOPS subunits, VPS11, VPS16, VPS18, VPS33, VPS39 and VPS41, respectively, were subjected to GSTpulldown assay using immobilized GST-RILP. 9E10 antibody was used for western-blotting to detect the protein bound to GST-RILP. The results revealed that RILP binds to all HOPS subunits except for VPS33, suggesting RILP interacts with HOPS complex. (B). GST-pulldown assay using GST-RILP, GST-RILP (1-198) and GST-RILP(199-401) demonstrated that N-terminal region (1-198aa) of RILP interacts with subunits of HOPS complex. Un-cropped blots/gels are presented in Supplementary Figure s7.

might potentially be mediated indirectly by Rab7 or by the RILP$\mathrm{Rab} 7$ complex. If this is the case, then the C-terminal region of RILP will be important for the interaction with HOPS subunits. We therefore next determined the region of RILP responsible for the interaction with HOPS subunits. Lysates derived from HeLa cells expressing the various HOPS subunits were processed for GSTpulldown assay using GST-RILP, GST-RILP(1-198) and GSTRILP(199-401). As shown in Figure 2B, all 5 interacting HOPS subunits were retained specifically by the immobilized N-terminal GST-RILP(1-198) (N-terminal region of RILP) but not the C-terminal GST-RILP(199-401) (C-terminal region of RILP) (Figure 2B). Furthermore, the retention of theses subunits by GST-RILP(1-198) is as efficient as that observed using GST-RILP. These results not only confirmed the interaction of HOPS subunits with RILP but also showed that the N-terminal region of RILP is responsible for the interaction, implying that the Rab7-interacting C-terminal region of RILP is not important for this interaction and that their interaction with RILP is likely independent of Rab7.

The interaction of HOPS subunits with RILP is independent of Rab7. To examine the possibility that interaction of HOPS subunits with RILP is independent of Rab7, we performed similar pull down assay using lysates derived from cells with Rab7 knockdown. As shown in Figure 3A, Rab7 protein levels were significantly reduced by its shRNA. When lysates derived from cells expressing various HOPS subunits with simultaneous Rab7 knockdown were used for the pull down assay, it was observed that their interaction with RILP occurred with comparable efficiency (Figure 3B), suggesting that significant reduction of Rab7 protein did not affect the interaction of HOPS subunits with RILP. To further corroborate this possibility, we tested the interaction between HOPS subunits and RILP(304AAA306), a mutant of RILP previously shown to have lost the ability to interact with Rab7 ${ }^{27}$. GST-pulldown experiments demonstrated that HOPS subunits interacted with RILP(304AAA306) with similar efficiency as observed for wild type RILP (Figure 3C). Under the similar condition, Rab7 was retained by RILP but not the RILP(304AAA306) mutant. Consistent with the observation that Rab7 was not able to recruit HOPS subunits to the membrane in co-expression assay, pull down assay using immobilized Rab7(GTP) detected only weak interaction with HOPS subunits and this weak interaction is not affected by mutation of L8A of $\mathrm{Rab7}^{27}$, which was previously shown to abolish Rab7 interaction with RILP (Figure 3D). Therefore, the weak interaction between Rab7 and HOPS subunit is independent of Rab7's ability to interact with RILP. Under the similar condition, RILP was efficiently retained by immobilized Rab7(GTP) but not Rab7L8A(GTP).

VPS41 is the major mediator for HOPS-RILP interaction. As described above, the robust interaction of 5 out of 6 HOPS subunits with RILP is independent of Rab7 and the weak interaction of HOPS subunits with Rab7 is independent of RILP. Previous studies indicated that Rab7 binds to HOPS complex through interaction with VPS39 and VPS41 $1^{8,30}$. We next investigated which HOPS subunit is most important for the observed interaction of RILP with 5 of the 6 subunits (VPS11, VPS16, VPS18, VPS39 and VPS41 but not VPS33).

To address whether RILP can recruit the endogenous HOPS complex, antibodies were used to label the subunits of HOPS, After testing various antibodies, we found that antibodies against VPS41 work for immuno-fluorescence assay. Endogenous VPS41 was present in diffuse cytoplasmic labeling with some small punctual structures in none transfected cells. Significant amount of endogenous VPS41 was recruited to the clustered RILP-labeled structures upon overexpression of RILP (Figure 4A).

To characterize which subunit is responsible for the recruitment of HOPS complex by RILP, small RNA interference assay was applied to silence each of the HOPS subunits and to examine the impact on the recruitment of other subunits onto RILP-marked vesicular structures (supplemental Figure s3). In this assay, VPS33 was not analyzed as it did not exhibit interaction in either recruitment or pull-down assays. We found that depleting VPS11, VPS16, VPS18 or VPS39 did not significantly affect the recruitment of remaining 4 subunits to RILP-positive structures (supplemental Figure s4). However depletion of VPS41 abolished the co-localization of VPS11, VPS16, VPS18 and VPS39 with RILP (Figure 4B).

Using the pull down assay, the retention of VPS11, VPS16, VPS18 and VPS39 by immobilized GST-RILP was significantly compromised when VPS41 was knocked down (Figure 4C and quantitative results were shown in Figure 4D), suggesting that VPS41 is the major player contributing to the interaction between RILP and HOPS complex, consistent with the results that RILP recruits endogenous VPS41.

VPS41 directly interact with RILP and the C-terminal half of VPS41 is responsible for interaction with RILP. Most subunits of the HOPS complex (except for VPS33) are big proteins with elongated structures, they can interact with each other easily, therefore, it is difficult to characterize the subunits that interact directly with RILP using the GST-pulldown or Co-IP assay from cell lysates. We tried to examine whether RILP can directly interact with VPS41 or not. GST-RILP immobilized onto GST sepharose beads was incubated with the purified His-VPS33 or His-VPS41, the bound proteins were examined through western-blot using His-tag antibody. The results demonstrated that RILP directly binds to VPS41, but not VPS33, (Figure 5A), indicating that RILP interacts with HOPS complex at least partly through direct binding to VPS41 subunit.

To begin to map the region of VPS41 responsible for the interaction with RLIP, we have created constructs expressing the N-terminal portion (residues 1-427aa) and C-terminal region (residues 

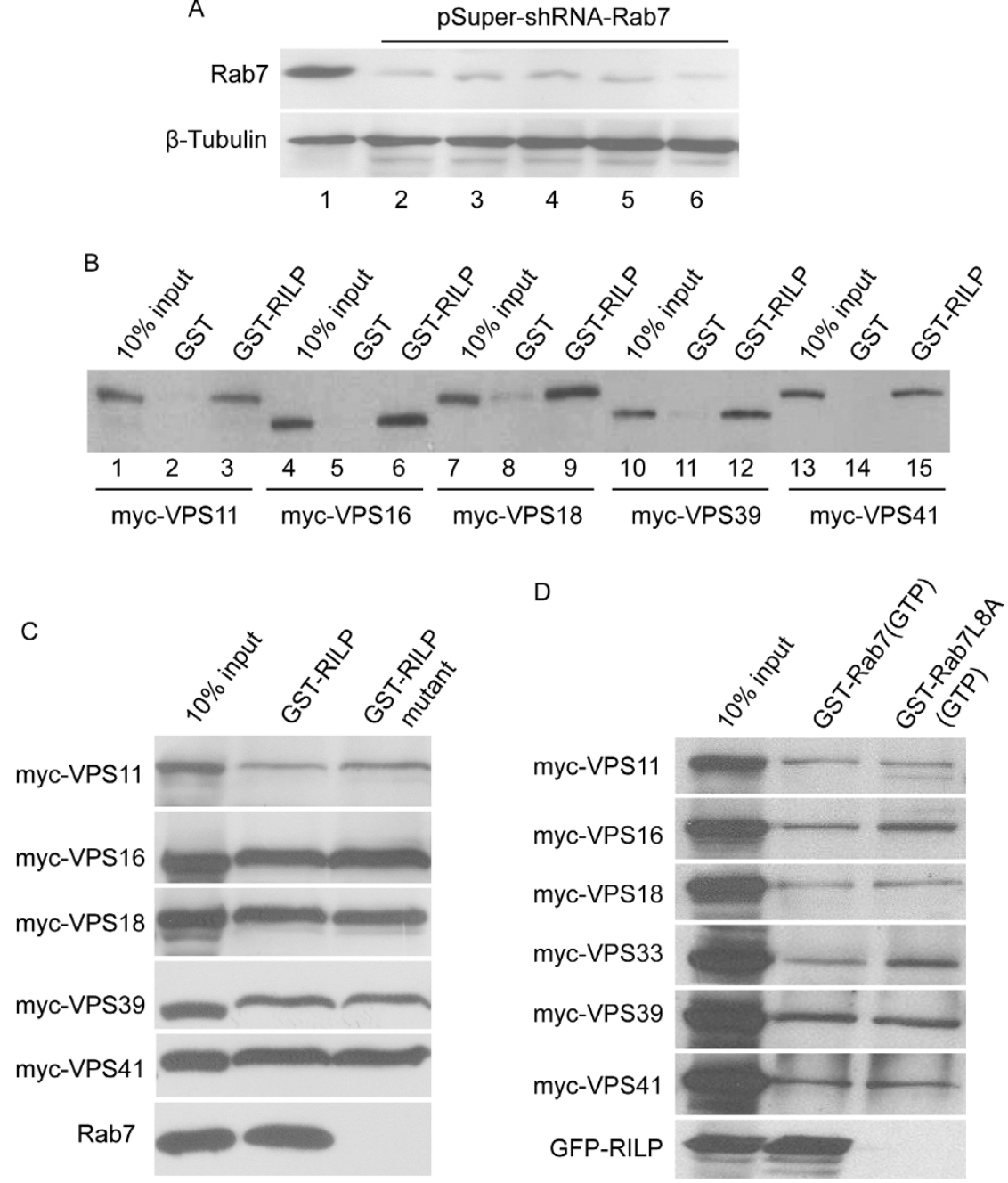

Figure 3 The interaction between RILP and HOPS complex is independent of Rab7. (A). Hela cells were transfected with pSuper.GFP-scrambleshRNA(lane 1) or shRNA-Rab7. 48 h later, knocked-down cells were transfected with myc-tagged VPS11 (lane 2), VPS16 (lane 3), VPS18 (lane 4), VPS39 (lane 5) and VPS41 (lane 6), respectively. $72 \mathrm{~h}$ later, cells were harvested and processed for detection of the knockdown efficiency. The results revealed that Rab7 was depleted efficiently in the cells expressing HOPS subunits. (B). Lysates derived from cells described above were subjected for GST-pulldown assay using GST-RILP, the results demonstrated that RILP can still bind to HOPS subunits when Rab7 was effectively depleted, suggesting RILP interacts with HOPS complex independent of Rab7. (C). Hela lysates containing myc-tagged VPS11, VPS16, VPS18, VPS39 and VPS41 were subjected for GSTpulldown assay using GST-RILP and GST-RILP304AAA306 mutant (defective in interacting with Rab7), the data showed that this RILP mutant can still bind to HOPS sununits, although no longer interacting with Rab7 (bottom panel). (D). Hela lysates containing myc-tagged VPS11, VPS16, VPS18, VPS33, VPS39 and VPS41 were subjected for GST-pulldown assay using GST-Rab7 or GST-Rab7L8A mutant (defective in interacting with RILP showed in bottom panel). Like wildtype Rab7, Rab7L8A can still weakly interacts with HOPS complex, suggesting Rab7 interacts with HOPS complex independent of RILP. Un-cropped blots/gels are presented in Supplementary Figure s8.

428-855aa) of VPS41. When co-expressed, Myc-tagged VPS41(428855) but not VSP41(1-427) was recruited to RILP-positive structures (Figure 5B). Consistently, GST-pulldown assay revealed that VPS41(428-855) but not VPS41(1-427) was retained by immobilized GST-RILP (Figure 5C), suggesting that the specific interaction of VPS41(428-855) with RILP is likely the basis for its selective recruitment onto the RILP-positive structures. Therefore, the C-terminal region of VPS41 mediates the interaction of HOPS complex with N-terminal region of RILP. Co- expression of C-terminal part of VPS41 with RILP induces the multiple and dispersed punctual structures, somewhat different from VPS41 wt and RILP together (Figure 1), suggesting the N-terminal part of VPS41 plays a role in regulating VPS41's function, although the underlying mechanisms need to be defined by further investigation.

HOPS complex regulates EGFR degradation. Both Rab7 and RILP are the interacting partners of HOPS complex, and are involved in
EGFR endocytosis, mediating ligand induced EGFR degradation ${ }^{5,29,31}$. However, the role of HOPS complex in regulating EGFR endocytosis is still not well established. Given the robust interaction of HOPS subunits with RILP and that VPS41 is primarily responsible for the interaction, we interfered the function of HOPS complex by depleting VPS41, using pSuper-mediated shRNA targeting VPS41 and then examined the EGF-stimulated EGFR internalization and degradation.

The protein level of cellular EGFR was monitored by westernblotting experiments. MCF7 cells were starved for over night. After stimulating with $10 \mathrm{ng} / \mathrm{ml}$ of EGF for the indicated times (Figure 6A and 6B), the cells were lysed for western-blot to detect the EGFR. The results demonstrated that depletion of VPS41 significantly inhibited the degradation of EGFR, as compared with control knockdown cells.

We next used immunofluorescence microscopy to monitor the degradation (disappearance) of endocytosed EGF-Rhodamine. The signals of EGF-Rhodamine were similar in control cells, VPS41knockdown cells, and VPS41(428-855) over-expressing cells after 


\section{A}

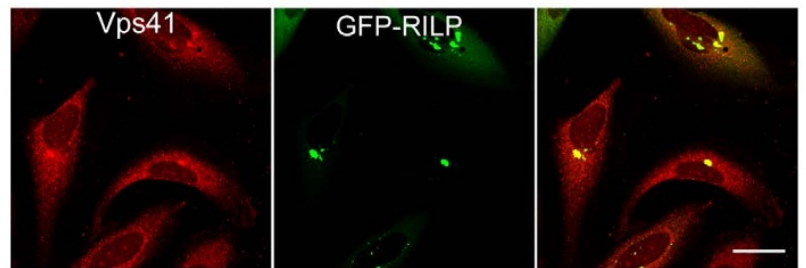

B
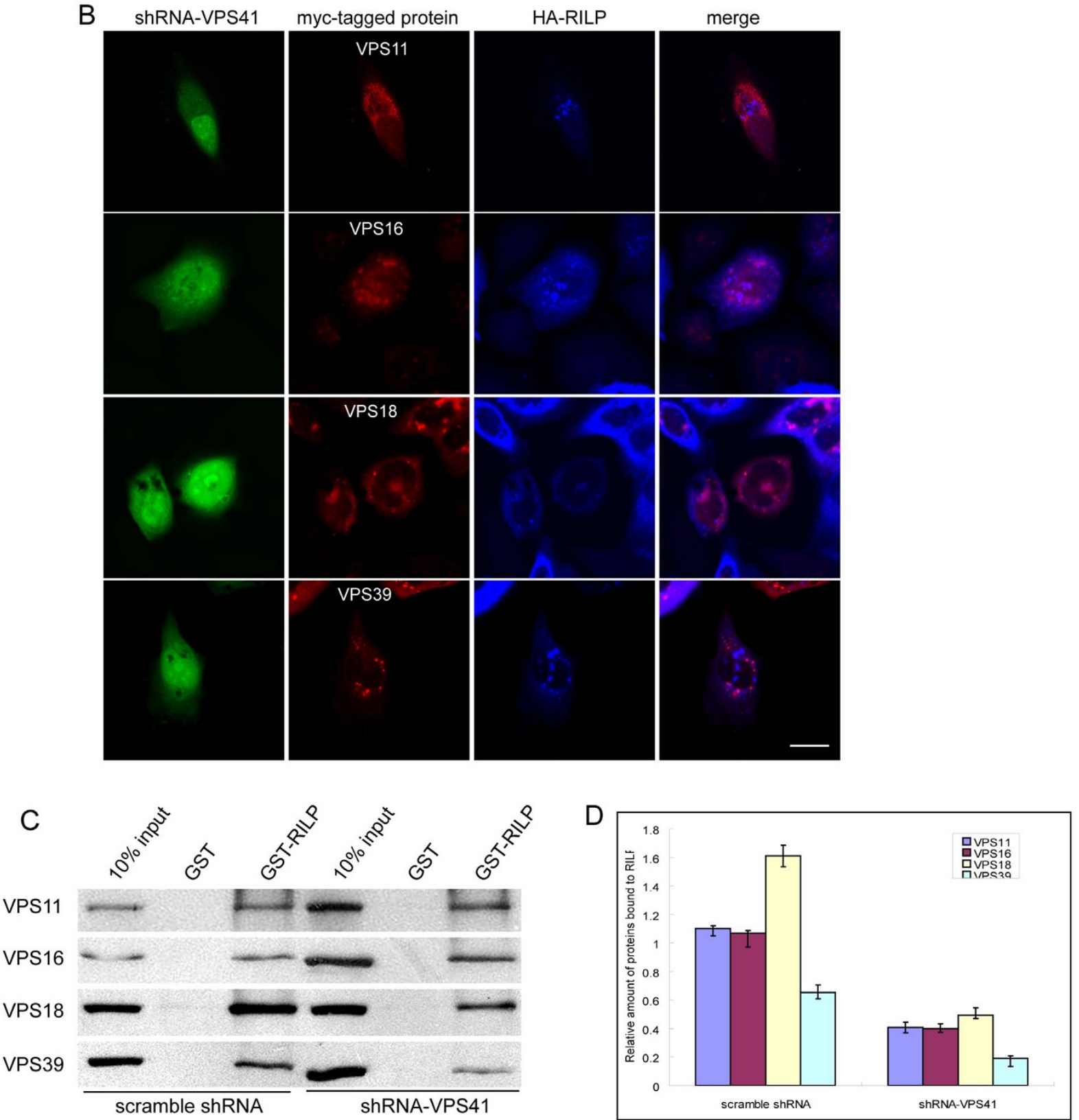

Figure $4 \mid$ VPS41 subunit plays a crucial role in RILP-HOPS interaction. (A). Hela cells expressing GFP-RILP were immuno-labeled with anti-VPS41 antibody to demonstrate the recruitment of endogenous VPS41 by RILP. (B). Hela cells were co-transfected with pSuper.GFP-shRNA-VPS41, myc-tagged HOPS subunits and HA-RILP, then processed for immuno-staining with myc and HA tag antibodies. Knockdown cells were revealed by co-expresed GFP signal. Myc-tagged proteins were revealed by Texas-red conjugated secondary antibodies and HA-tagged proteins were revealed by Cy5conjugated secondary antibodies. Immuofluorescence microscopy showed that depletion of VPS41 disrupts the co-localization of other HOPS subunits with RILP. (C). GST-pulldown experiments demonstrated that depletion of VPS41 decreases the amount of HOPS subunits bound to RILP. (D). Quantitative analysis of the results from triplicate experiments revealed that the amount of HOPS subunits bound to RILP is significantly decreased when VPS41 was knocked-down. Un-cropped blots/gels are presented in Supplementary Figure s9. bar $=20 \mu \mathrm{m}$. 


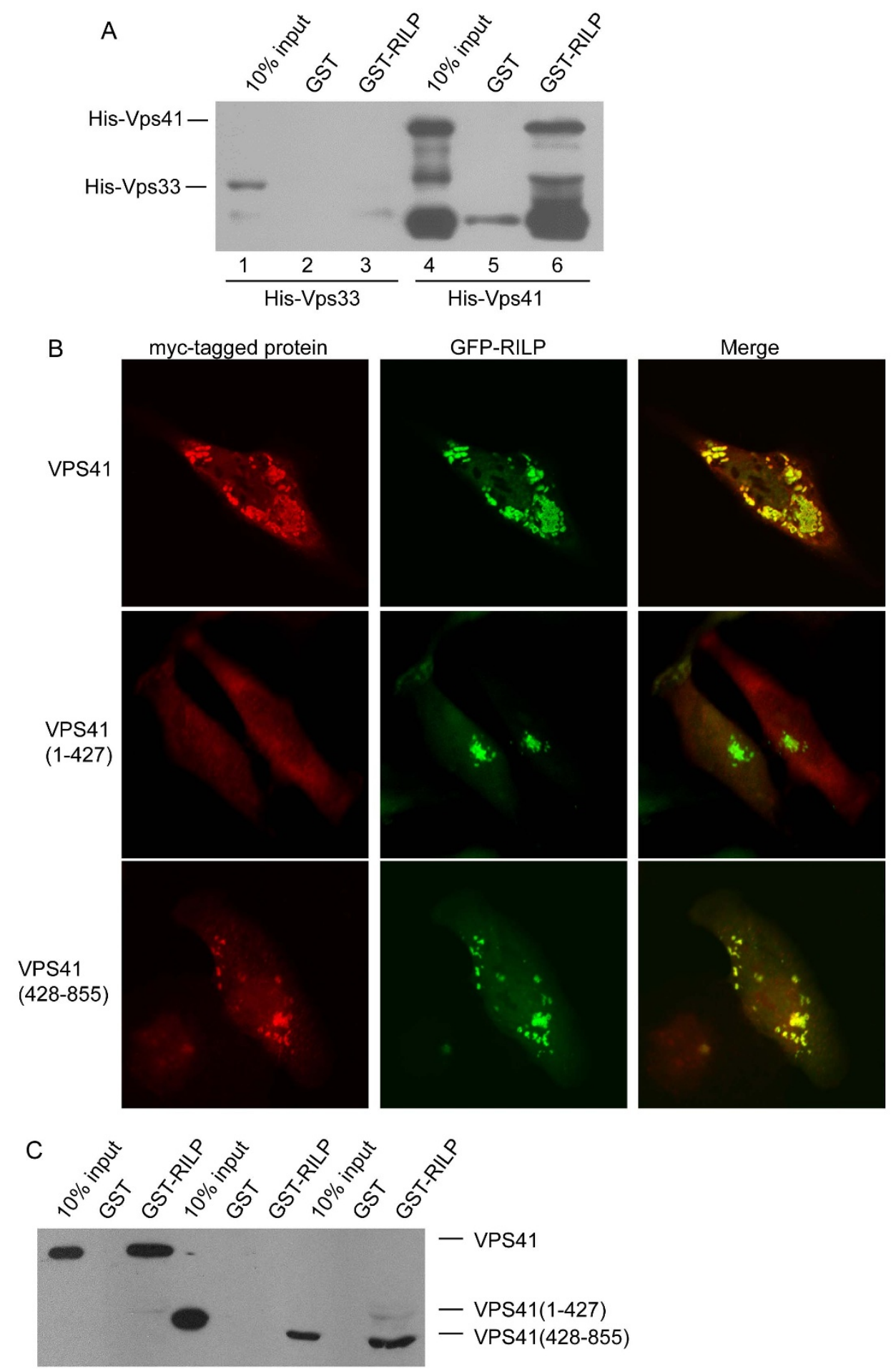

Figure 5 | C-terminal region of VPS41 contributes to the interaction between RILP and HOPS complex. (A). Purified His-VPS41 or His-Vps33 recombinant protein was incubated with immobilized GST-RILP to show RILP directly interacts with VPS41, but not VPS33. (B). Hela cells were coexpressed with GFP-RILP and myc-VPS41, myc-VPS41(1-427) or VPS41(428-855), respectively. Immunofluorescence microscopy revealed that RILP can recruit wild type VPS41 and VPS41(428-855), but not VPS41(1-427). (C). Pulldown experiments showed that RILP specifically binds to VPS41 through its C-terminal region(428-855aa). Un-cropped blots/gels are presented in Supplementary Figure s10. Bar $=20 \mu \mathrm{m}$.

short time of endocytosis (5 min) (Fig s5). VPS41(428-855) is expected to act as a dominant negative mutant via competing with endogenous VPS41 for ineraction with RILP and its effect on EGF degradation was evaluated along with VPS41 silence. After endocytosis and degradation of 30 and $60 \mathrm{~min}$, the signals of EGFRhodamine were represented with few fine vesicular labeling in control cells (Figure 6C). However, numerous vesicular structures were intensively labeled with EGF-Rhodamine in VPS41-knocked down cells even after $60 \mathrm{~min}$ of endocytosis and degradation (Figure 6D), suggesting a failure of endocytosed EGF-Rhodamine to be delivered to the degradative lysosomes. Since significant number of the labeled structures did not colocalize with EEA1 (an early endosomal marker), endocytosis and transit through the early endosome are likely not affected by VPS41 knockdown. These structures 
A
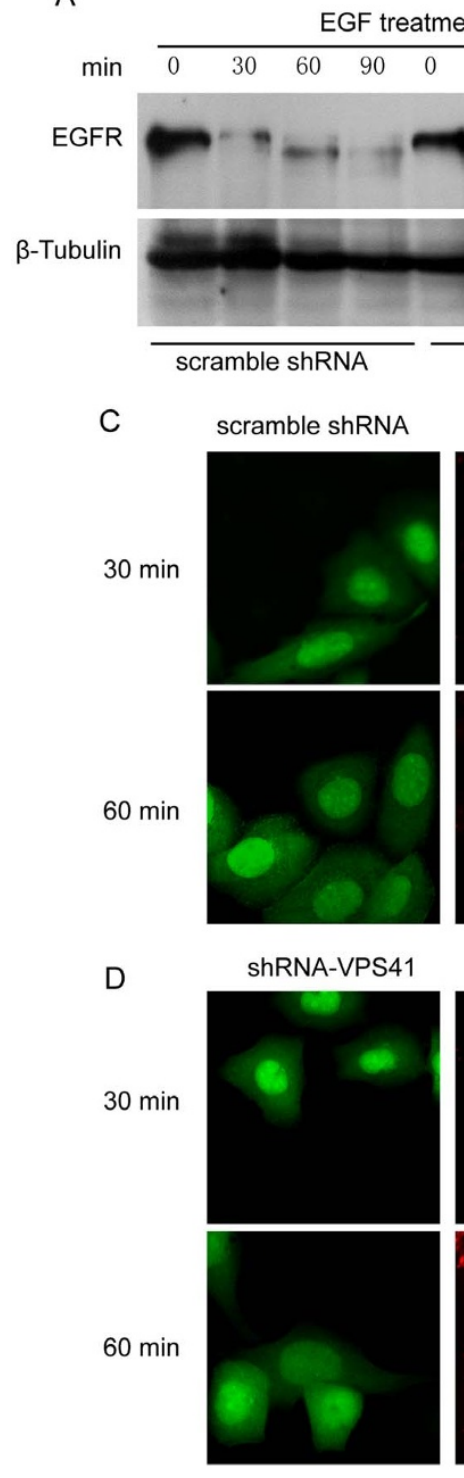

E

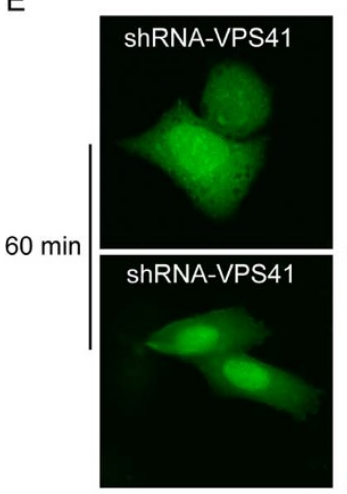

B

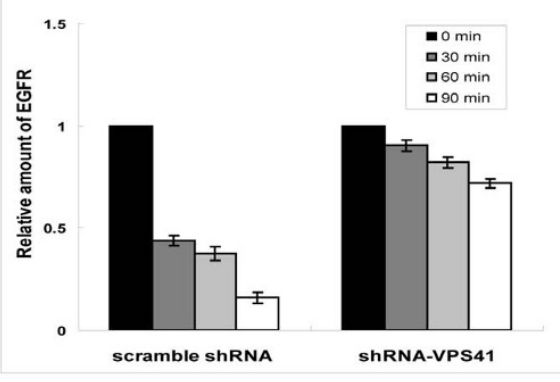

EEA1

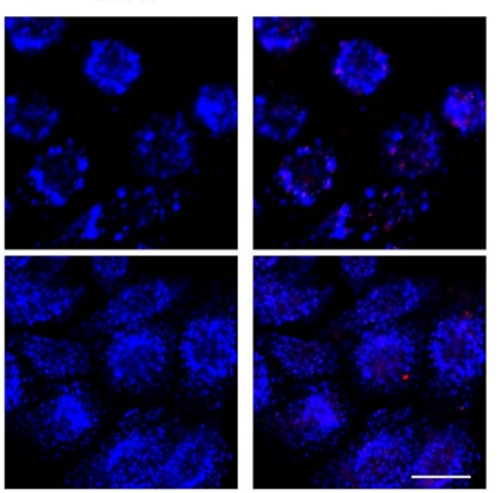

EEA1
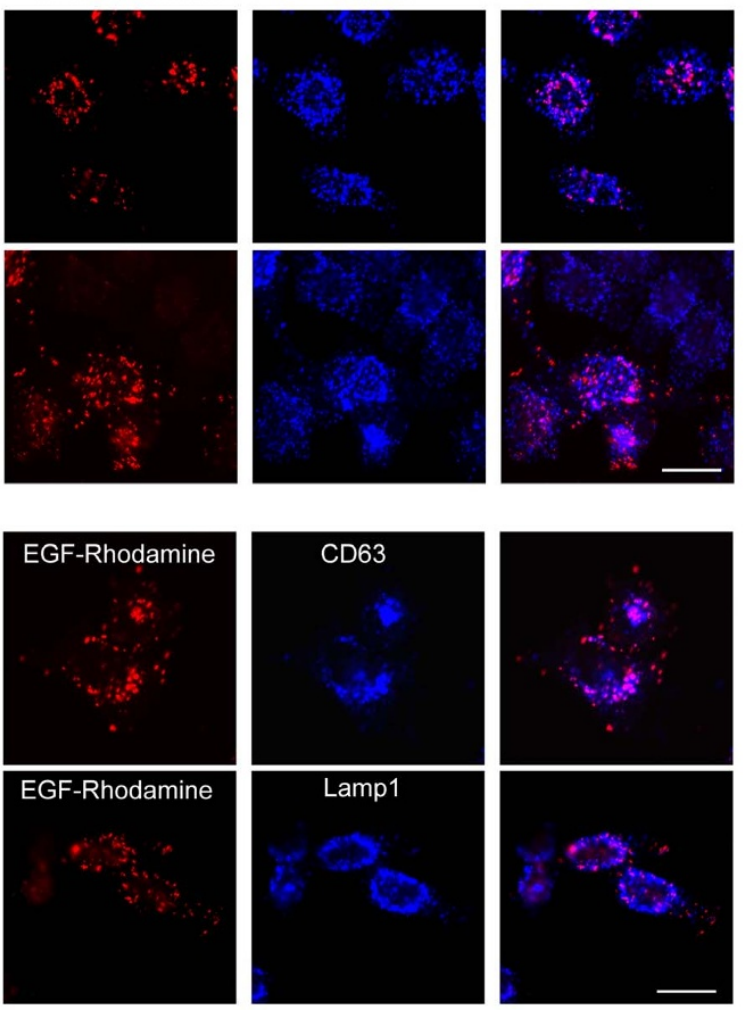
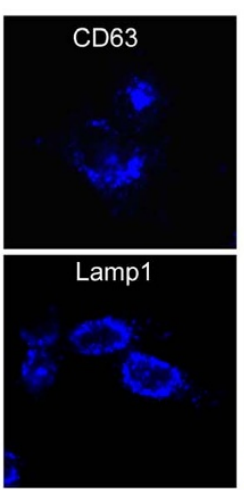

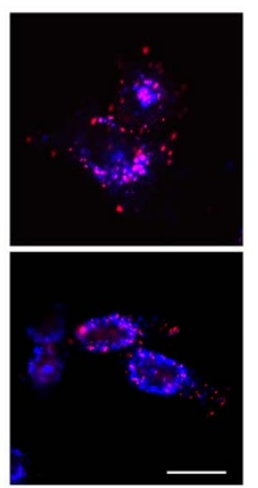

Figure 6 Disruption of HOPS complex inhibits EGFR degradation. (A). MCF7 cells were transfected with pSuper.GFP-scramble-shRNA or shRNAVPS41, $48 \mathrm{~h}$ later, the cells were starved for overnight, then stimulated with EGF for the indicated times. The protein level of EGFR was examined by western-blot using $\mathrm{mAb}$ against EGFR. The results demonstrated EGFR decreased quickly in scramble knocked-down cells, but the degradation of EGFR is compromised in shRNA-VPS41 knocked-down cells. Un-cropped blots/gels are presented in Supplementary Figure s11. (B). Quantitative analysis from 3 independent experiments reveals that EGFR degradation is significantly arrrested in shRNA-VPS41 knocked-down cells. (C). Immunofluorescence microscopy showed rapid endocytic clearance of endocytosed EGF-Rhodamine in scramble knockdown cells. (D). depletion of VPS41 delayed clearance of endocytosed EGF-Rhodamine as evidenced by the accumulation of endocytosed EGF-Rhodamine in structures partially overlapping with EEA1labeled early endosome after $60 \mathrm{~min}$ internalization. (E). EGF-Rhodamine accumulated vesicles were co-localized with late endosomal/lysosomal markers CD63 and Lamp1 after 60 min of endocytosis in cells with VPS41 depletion. Bar $=20 \mu \mathrm{m}$. 
A
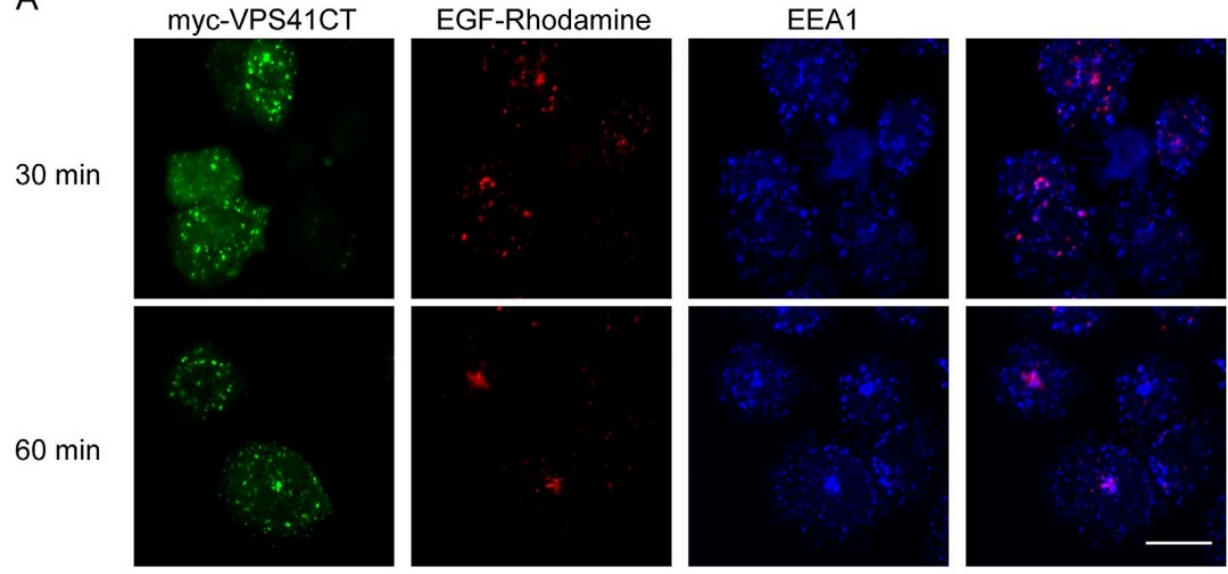

B
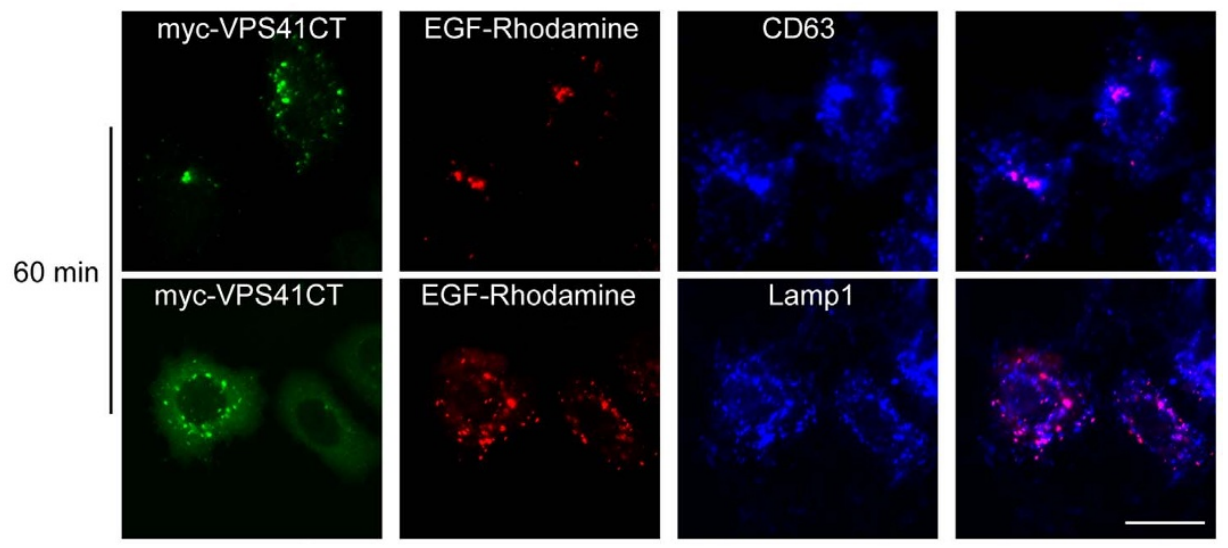

Figure $7 \mid$ The effects of overexpressing VPS41(428-855) (VPS41CT) on EGF-Rhodamine endocytosis and degradation. (A). over-expressing VPS41CT delayed endocytic clearance of EGF-Rhodamine similar to the effect of shRNA-VPS41. (B). EGF-Rhodamine accumulated vesicles were colocalized with late endosomal/lysosomal markers CD63 and Lamp1 after 60 min endocytosis in cells expressing VPS41CT. Bar $=20 \mu \mathrm{m}$.

were more colocalized with late endosomal and lysosomal markers CD63 and Lamp1 after 60 min of endocytosis (Figure 6E), suggesting an arrest of endocytosed EGF-Rhodamine in late endosomes and lysosomes. Since VPS41 knockdown reduced interaction of RILP with HOPS complex, the observed results could be explained by proposing that the interaction of RILP with HOPS is important for endocytic trafficking of endocytosed EGF. We explored this possibility further. Since the C-terminal region of VPS41 is responsible for interaction with RILP, overexpression of the C-terminal region of VPS41 is expected to compete with endogenous VPS41 (and therefore HOPS complex) with RILP. We therefore examined the consequence of overexpression of VPS41(428-855) (VPS41CT). As shown in Figure 7A over-expression of VPS41CT also caused accumulation of EGF-Rhodamine in vesicular structures, and these structures were more co-localized with late endosomal/lysosomal markers CD63/Lamp1 (Figure 7B). Therefore disruption of interaction of endogenous RILP with VPS41 (and the HOPS complex) caused delayed degradation of endocytosed EGF-Rhodamine, suggesting the interaction of RILP with VPS41 is functionally important for endocytic trafficking of endocytosed EGF to the lysosome.

\section{Discussion}

Rab7 plays major roles in regulating late endolysosomal trafficking, which is governed by its two major partners HOPS and RILP ${ }^{4,5,32-34}$. Nevertheless, there is no RILP ortholog in lower eukaryotic cells, such as yeast cell, thus the mechanism for the endolysosomal membrane trafficking regulated by HOPS, Rab7 and RILP in mammalian system might exhibit difference due to the presence of RILP and its two related proteins ${ }^{25}$. In our present study, we have found the inter- action of RILP with HOPS complex that is independent of Rab7, which offers a novel regulatory mechanism for Rab7/HOPS in late endolysosomal trafficking.

The subunit interaction within the HOPS complex has been examined $^{7}$. VPS11, VPS16, VPS18 and VPS33 may interact to form the core complex, with VPS33 interacting with SNARE proteins, VPS11 and VPS18 associating with VPS39 and VPS41, respectively. HOPS complex targeting to the vesicle is proposed to be mediated through VPS39/VPS41 interaction with Rab7 to modulate membrane fusion $^{8,29}$ (schematically depicted in Figure 8A). The activity of yeast Vps41p is also regulated by Yck3 kinase and Gyp7p (GAP, GTPase activating protein $)^{29,30,35}$. VPS39 serves as GEF for Rab7/Ypt7p in yeast ${ }^{8}$. Over-expression of RILP induces enlarged and clustered late endosome/lysosome, suggesting RILP interacting with Rab7 may stabilize the association of Rab7 on the membrane, thus promoting membrane fusion and positioning them to the peri-Golgi region ${ }^{25}$ due to interaction with dynein motor $^{28}$. Although expression of Vam6 (human VPS39) also induces enlarged late endosome/lysosome, it was not addressed whether this is linked to Rab7 or RILP ${ }^{23}$. We have therefore investigated the relationship between HOPS and RILP. Interestingly, we observed the robust interaction of VPS11, VPS16, VPS18, VPS39 and VPS41 subunits with RILP as examined by membrane recruitment in vivo and GST-pull down in vitro. The $3 \mathrm{D}$ structure analysis showed VPS33 interacts with VPS16 only ${ }^{36}$, and subunits organization investigation also demonstrated that each subunit interacts with at least 2 other subunits except for VPS337,37, therefore, RILP may interact with VPS33 more indirectly or weakly through interacting with VPS41, thus the interaction between VPS33 and RILP was not observed in our GST-RILP pulldown experiments. 


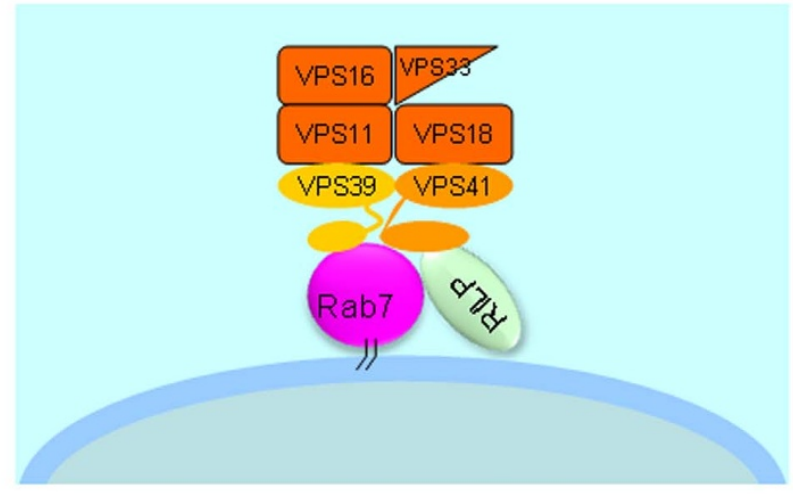

B

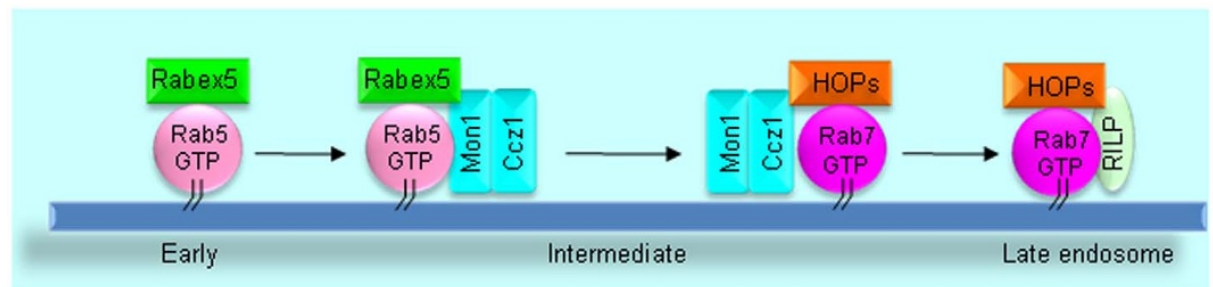

Figure $8 \mid$ A working model of HOPS-Rab7-RILP interaction on the endosomal membrane. In this model, Rab7 interacts with both accessory subunits VPS39 and VPS41 of HOPS complex, while RILP interacts with Rab7 and VPS41 to stabilze Rab7-HOPS at the endosomal membrane. (B). a working model of Rab7 modulating endosomal membrane transition regulated by Mon1/Ccz1, HOPS and RILP. In this model, Mon1-Ccz1 plays cis-regulation to exclude Rab5 and recruit HOPS complex, activating Rab7, while RILP plays trans-regulation to stabilize Rab7-HOPS complex onto late endosomal membrane.

Several lines of evidence suggest that the interaction of HOPS subunits with RILP is independent of Rab7. Firstly, the C-terminal region of RILP is responsible for interaction with Rab7. However, its interaction with HOPS subunits was mapped to the N-terminal region, demonstrating different and not overlapping regions of RILP are involved in the interaction with HOPS complex and Rab7, respectively. Secondly, interaction of HOPS complex with RILP was not significantly affected when expression of Rab7 protein was significantly knocked down. Thirdly, we have previously resolved the x-ray crystal structure of RILP-Rab7 complex and this structural information has allowed us to mutate the key residues of RILP involved in the interaction with Rab7 to create mutants of RILP that are no longer able to interact with Rab7. One such mutant RILP(304AAA306) was tested and it exhibited similar and robust interaction with HOPS complex. The interaction of HOPS subunits with Rab7(GTP) was also observed but the interaction is much weaker than that observed between RILP and HOPS. Furthermore, the interaction of HOPS with Rab7 is also not dependent on Rab7's ability to interact with RILP, as a mutant of Rab7 incapable of RILP interaction exhibited similar weak interaction with HOPS.

It is well established that the C-termianl end of RILP interacts with Rab7, while the N-terminal end of RILP interacts with dynein/dynactin to drive vesicle movement $\mathrm{t}^{6,27,28,38}$. Our results revealed that the $\mathrm{N}$ terminal region of RILP is also involved in the interaction with HOPS complex. At the present, we are not clear whether the interaction of RILP with HOPS and dynein/dynactin is mutually exclusive or could occur simultaneously.

Since 5 subunits of HOPS complex were shown to interact with RILP, we have attempted to define which subunit is most directly involved. Since recombinant subunits were not successfully produced, we were not able to investigate direct interaction of RILP with each of the HOPS subunits. We therefore resorted to another approach of silencing each of the subunits to see the impact on membrane recruitment of the rest and we were able to demonstrate that VPS41 is most crucially involved in membrane recruitment of
HOPS complex in response to RILP overexpression and its silence caused failure of recruitment of other 4 subunits to the RILP structures, while silence of all other subunits had no major impact. Furthermore, knockdown of VPS41 also significantly reduced the interaction of other subunits with RILP in the GST-pull down assay. We also examined whether the stability of HOPS complex is affected by loss of VPS41. Since there are no good antibodies commercially available capable of detecting endogenous proteins efficiently, myctagged VPS11, VPS18, VPS33 and VPS39 were transfected into MCF7 cells stably expressing shRNA-VPS41, respectively. The resulting cell lysates were processed for immuno-precipitation assay using antibody against VPS16 that works for immunoprecipiation. The data revealed that these proteins (especially VPS11 and VPS39) bound to VPS16 decreased upon depletion of VPS41 (Figure s6). This data to some extent suggests that VPS41 may be important for stabilizing the HOPS complex.

In addition, we have also provided evidence that the C-terminal region of VPS41 is responsible for interacting with RILP. A ringstructure in the C-terminal region of human VPS41 is divergent from yeast VPS41 and this divergence may facilitate the interaction with mammalian specific regulators such as RILP, further suggesting the specific and unique mechanism for regulating endosomal trafficking in mammalian cells.

The functional relevance of the RILP interaction with HOPS complex was shown by following the endocytic degradation of EGFR as assessed by western blot and EGF-Rhodamine as assessed by immunofluorescence. When VPS41 was knocked down, EGF induced degradation of EGFR was compromised significantly, and the degradation of endocytosed EGF-Rhodamine was greatly retarded upon VPS41 knockdown. Furthermore, overexpression of the C-terminal region of VPS41 to prevent interaction of endogenous HOPS with RILP also caused retardation of degradation of endocytosed EGFRhodamine.

Based on these results, we are proposing a working model of dual interaction of HOPS complex with both Rab7 and RILP. Whether 
HOPS complex is involved in GEF activity of Rab7 is yet to be defined, but Rab7 interaction with RILP will lead to endosomal recruitment of Rab7-RILP complex. Through robust interaction of C-terminal region of VPS41 with RILP, the HOPS complex is recruited to the Rab7-RILP complex, enabling the weak interaction between HOPS complex and Rab7 to occur to form a stable HOPSRab7-RILP complex (Figure 8A), which will be intimately involved in endocytic trafficking. In this model, HOPS complex can be also considered as a downstream effector of Rab7-RILP complex in addition to the possibility that HOPS complex may still act as an upstream activator of Rab7 in view of yeast study.

Recent studies found that Mon1-Ccz1 protein can inactivate Rab5 through displacing Rabex5 and activate Rab7 through interaction with HOPS complex to regulate early-to-late endosomal membrane transition ${ }^{14,15,39-41}$. In view of our results, we hypothesis a regulatory model in Figure 8B, in which Mon1-Ccz1 plays cis-regulation for HOPS, while RILP plays trans-regulation for HOPS, and the activity and stabilization on membrane of Rab7 are controlled by HOPS complex, RILP and Mon1-Ccz1.

Another complex COVERT (class C core vacuole/endosome tethering) was identified in yeast, sharing the class $\mathrm{C}$ core complex with HOPS complex, with VPS39 and VPS41 being substituted by VPS3 and VPS8, respectively ${ }^{42}$. COVERT may modulate the activity of Rab5, but its function on Rab7/Ypt7p is not clear ${ }^{43}$. So far, the mammalian COVERT has not been characterized completely, let alone the relation to RILP. Further studies may help to uncover novel regulation mechanisms in mammalian cells.

\section{Methods}

Antibodies. The monoclonal antibodies (mAbs) against EEA1 and EGFR were from BD (BD Biosciences, Palo Alto, CA); mAbs against human Lamp1, CD63 and HA-tag were obtained from the Developmental Studies Hybridoma Bank maintained by the University of Iowa (Department of Biological Science, Iowa City, IA, USA). mAbs against Rab7 and $\beta$-tubulin were from Sigma (Aldrich, St Louis MO, USA). mAb against EGFP were purchased from the Clontech Laboratories, BD Biosciences (Palo, Alto, CA, USA). mAb against VPS16 (which works in immunoprecipiataion) and anti-VPS41 (which works in immunofluorescence) were purchased from Santa Cruz Biotechnology (Dallas, Texas, USA). Polyclonal anti-myc tag antibody was purchased from Millipore (Billerica, Massachusetts, USA). mAb against Myc-tag (9E10) was obtained from American Type Culture Collection (ATCC, Manassas, VA 20108, USA). mAb against His-tag was from Transgen Biotech (Beijing,China). HRPconjugated secondary antibodies were purchased from Pierce (Rockford, IL, USA). Texas red- or Cy5-conjugated secondary antibodies were from Jackson ImmunoResearch (West Grove, PA, USA).

Expression constructs. Myc-tagged VPS11, VPS16, VPS18, VPS33, VPS39 and VPS41 expression constructs were generated by subcloning the respective human VPS coding region into pCMV-Myc vector. His-VPS41 and His-VPS33 expression plasmids were generated by subcloning VPS41 or VPS33 coding region into pET28a vector. GFP-RILP expression construct was described previously ${ }^{44}$. HA-RILP was generated by subcloning the coding region of RILP cDNA into EcoR1/Not1 sites of pDHA-neo vector. GST-RILP, GST-RILP(1-198), GST-RILP(199-401)were described previously ${ }^{23}$. Myc-RILP(304AAA306) and Rab7L8A constructs were described previously ${ }^{25}$. GST-RILP(304AAA306) and GST-Rab7L8A constructs were generated by subcloning RILP(304AAA306) and Rab7L8A into pGEX-4T-1 vector. The primers (5-AATCTCGAGATGGCGGAAGCAGAGGAGC AG-3 and 5CTACGCGGCCGCCTATGCTGCATTTTTCCC-3) and the primers (5AATCTCGAGATGCTCTGGGAATATGAAGTT-3 and 5-CTACGCGGCCGC CTATTTTTTCAT CTCCAA-3) were used to amplify the coding regions of Nterminal fragment 1-427aa and C-terminal fragment 428-855aa of VPS41, respectively. The fragments were sub-cloned into Xho I/Not I sites of pDmyc vector to generate Myc-VPS41(1-427) and myc-VPS41(428-855), respectively.

Cell culture and transfection. HeLa and MCF7 cells were cultured in RPMI 1640 medium supplemented with $10 \%$ fetal bovine serum (HyClone) in a $5 \% \mathrm{CO}_{2}$ incubator at $37^{\circ} \mathrm{C}$. Transient transfection of plasmids was conducted using TurboFect in vitro transfection reagent (Thermo, Massachusetts, USA) according to the manufacturer's protocol.

immunofluorescence microscopy. Immuno-staining was performed as described ${ }^{45}$. Briefly, cells grown on coverglasses were washed with PBSCM (PBS containing $1 \mathrm{mM}$ $\mathrm{CaCl}_{2}$ and $1 \mathrm{mM} \mathrm{MgCl}$ ) and then fixed with $3 \%$ paraformaldehyde in PBSCM at $4{ }^{\circ} \mathrm{C}$. After sequential washing with PBSCM supplemented with $50 \mathrm{mM} \mathrm{NH}_{4} \mathrm{Cl}$, cells were permeabilized with $0.1 \%$ saponin (Sigma, St. Louis, MO, USA) in PBSCM for $15 \mathrm{~min}$ at room temperature, and were subjected for immuno-staining using the antibodies indicated. Immuno-labeled cells or/and GFP-expressing cells were analysed by using confocal immunofluorescence microscopy equipped with Carl Zeiss LSM5 EXITER laser (Zeiss, Jena, Germany).

GST Pull-down experiment, western blot, in vitro bingding assay and immunoprecipitation. GST-pulldown assay was performed as described ${ }^{45}$. Briefly, HeLa cells were transfected with myc-tagged constructs as indicated in the text. After $18 \mathrm{~h}$, cells were harvested and lysed in the binding buffer (containing $20 \mathrm{mM} \mathrm{HEPES,} \mathrm{pH} \mathrm{7.4,}$ $100 \mathrm{mM} \mathrm{NaCl}, 5 \mathrm{mM} \mathrm{MgCl} 2,1 \% \mathrm{TX}-100$, and EDTA-free proteinase inhibitor cocktail from Roche (Nutley, NJ, USA)) for 1 hour at $4{ }^{\circ} \mathrm{C}$. The lysates were spun down, and the supernatant was subjected for GST-pulldown assay by using the indicated GST-fusion proteins coupled to the GST-Sepharose 4B resin (GE healthcare, Fairfield, Connecticut, USA). The bound proteins were resolved by SDSPAGE, transferred to nitrocellulose filter, and detected by western-blot assay using ECL system (Pierce, Rockford, IL, USA).

For direct binding assay, His-Vps41 or His-Vps33 was expressed in E. coli BL21(DE3) and purified by using HIS-Select Nickel Affinity Gel (Sigma) according to the manufacturer's instructions. $1 \mu$ g purified His-VPS41 or His-Vps33 recombinant protein was diluted in $500 \mu \mathrm{l}$ 5\% BSA in GST binding buffer (PBS containing $1 \mathrm{mM}$ $\mathrm{MgCl}_{2}, 1 \mathrm{mM}$ DTT), respecitvely, then incubated with immobilized GST-RILP as described above. After extensive washing, the retained proteins by GST-RILP were detected by western-blot using anti-His tag antibody.

For immuno-precipitation, HeLa cells were transfected with the indicated expression plasmids, respectively. After 18 hours, the cells were harvested and lysed in lysis buffer ( $100 \mathrm{mM} \mathrm{NaCl}, 5 \mathrm{mM} \mathrm{MgCl}_{2}, 1 \%$ TX-100, $20 \mathrm{mM}$ Hepes, $\left.\mathrm{pH} 7.2\right)$ on ice for 1 hour, and the cell lysates were spun down and subjected for immunoprecipitation by incubating with polyclonal myc tag antibodies coupled to protein A agarose beads. The bound proteins were analyzed by western-blot.

pSuper-mediated small hairpin RNA interference. pSuper.GFP-shRNA-Rab7 was described recently ${ }^{46}$. Targeting sequences for VPS11 (5'GCCTACAAACTACGGGTGA3'), VPS16 (5'GCTGACAAGATTCAACGGG 3'), VPS18 (5'GCGCATTACCAGTCTTGTC3'), VPS33 (5'GGAGTACAGCTTAGATCTC $3^{\prime}$ ), VPS39 (5'AGTGCTATCTCCATACAAA3'), VPS41 (5'GGGATTTGCCAAGTCGATA $\left.3^{\prime}\right)$ were constructed into pSuper.GFP.neo vector according to the manufacturer's instruction, respectively, to express shRNA. For these shRNA targets, they were chosen based on their efficient knockdown of target gene expression in trial experiments using several targets for each gene. For control knockdown, sequence (5'GATGCAACCACCCACGAAT3') was used for scramble-shRNA. The knockdown effects were examined 72 hours after transfection of the shRNA expressing construct.

EGFR degradation experiments. EGFR degradation experiments were carried out as described $^{4,44}$. Briefly, cells were starved in starvation medium (RPMI containing $50 \mathrm{mM}$ Hepes and 1\% BSA) for overnight. After extensively wash with starvation medium, the cells were stimulated with EGF $(10 \mathrm{ng} / \mathrm{ml})$ in starvation medium at $37^{\circ} \mathrm{C}$ for the indicated time. The protein level of EGFR was assessed by immuno-blotting assay.

For immuno-fluorescence microscopy analysis of EGFR endocytosis, cells grown on coverslips were starved and incubated with $0.2 \mu \mathrm{g} / \mathrm{ml}$ Rhodamine-conjugated EGF in starvation medium at $4^{\circ} \mathrm{C}$ for 1 hour, then processed for endocytosis at $37^{\circ} \mathrm{C}$ in complete medium. After internalization, cells were fixed and analysed by using confocal immuno-fluorescence microscope.

1. Bucci, C., Thomsen, P., Nicoziani, P., McCarthy, J. \& van Deurs, B. Rab7: a key to lysosome biogenesis. Mol. Biol. Cell 11, 467-480 (2000).

2. Ceresa, B. P. \& Bahr, S. J. Rab7 activity affects epidermal growth factor : epidermal growth factor receptor degradation by regulating endocytic trafficking from the late endosome. J.Biol.Chem. 281, 1099-1106 (2006).

3. Nickerson, D. P., Brett, C. L. \& Merz, A. J. Vps-C complexes: gatekeepers of endolysosomal traffic. Curr.Opin.Cell.Biol. 21, 543-551 (2009).

4. Wang, T., Zhang, M., Wu, X. \& Hong, W. Rab7: Role of its protein interaction cascades in endo-lysosomal traffic. Cell.Signal. 23, 516-521 (2011).

5. Cantalupo, G., Alifano, P., Roberti, V., Bruni, C. B. \& Bucci, C. Rab-interacting lysosomal protein (RILP): the Rab7 effector required for transport to lysosomes. EMBO J. 20, 683-693 (2001).

6. Jordens, I. et al. The Rab7 effector protein RILP controls lysosomal transport by inducing the recruitment of dynein-dynactin motors. Curr.Biol. 11, 1680-1685 (2001).

7. Plemel, R. L. et al. Subunit organization and Rab interactions of Vps-C protein complexes that control endolysosomal membrane traffic. Mol.Biol.Cell 22, 1353-1363 (2011).

8. Wurmser, A. E., Sato, T. K. \& Emr, S. D. New component of the vacuolar class CVps complex couples nucleotide exchange on the Ypt7 GTPase to SNAREdependent docking and fusion. J.Cell Biol. 151, 551-562 (2000).

9. Seals, D. F., Eitzen, G., Margolis, N., Wickner, W. T. \& Price, A. A Ypt/Rab effector complex containing the Secl homolog Vps33p is required for homotypic vacuole fusion. Proc.Natl. Acad.Sci U S A 97, 9402-9407 (2000).

10. Sato, T. K., Rehling, P., Peterson, M. R. \& Emr, S. D. Class C Vps protein complex regulates vacuolar SNARE pairing and is required for vesicle docking/fusion. Mol.Cell 6, 661-671 (2000). 
11. Preston, R. A., Reinagel, P. S. \& Jones, E. W. Genes required for vacuolar acidity in Saccharomyces cerevisiae. Genetics 131, 551-558 (1992).

12. Peterson, M. R. \& Emr, S. D. The class C Vps complex functions at multiple stages of the vacuolar transport pathway. Traffic 2, 476-486 (2001).

13. Peralta, E. R., Martin, B. C. \& Edinger, A. L. Differential effects of TBC1D15 and mammalian Vps39 on Rab7 activation state, lysosomal morphology, and growth factor dependence. J.Biol.Chem. 285, 16814-16821 (2010).

14. Bohdanowicz, M. \& Grinstein, S. Vesicular traffic: a Rab SANDwich. Curr.Biol. 20, R311-314 (2010)

15. Poteryaev, D., Datta, S., Ackema, K., Zerial, M. \& Spang, A. Identification of the switch in early-to-late endosome transition. Cell 141, 497-508 (2010).

16. Maldonado, E., Hernandez, F., Lozano, C., Castro, M. E. \& Navarro, R. E. The zebrafish mutant vps18 as a model for vesicle-traffic related hypopigmentation diseases. Pigment Cell Res. 19, 315-326 (2006).

17. Pulipparacharuvil, S. et al. Drosophila Vps16A is required for trafficking to lysosomes and biogenesis of pigment granules. J. Cell Sci. 118, 3663-3673 (2005).

18. Schonthaler, H. B. et al. The zebrafish mutant lbk/vam6 resembles human multisystemic disorders caused by aberrant trafficking of endosomal vesicles. Development 135, 387-399 (2008).

19. Yu, J. F., Fukamachi, S., Mitani, H., Hori, H. \& Kanamori, A. Reduced expression of vps 11 causes less pigmentation in medaka, Oryzias latipes. Pigment. Cell Res. 19, 628-634 (2006)

20. Suzuki, T. et al. The mouse organellar biogenesis mutant buff results from a mutation in Vps33a, a homologue of yeast vps33 and Drosophila carnation. Proc Natl Acad Sci U S A. 100, 1146-50 (2003).

21. Chintala, S. et al. The Vps33a gene regulates behavior and cerebellar Purkinje cell number. Brain Res. 1266, 18-28 (2009).

22. Gissen, P. et al. Mutations in VPS33B, encoding a regulator of SNARE-dependent membrane fusion, cause arthrogryposis-renal dysfunction-cholestasis (ARC)syndrome. Nat. Genet. 36, 400-404 (2004).

23. Caplan, S., Hartnell, L. M., Aguilar, R. C., Naslavsky, N. \& Bonifacino, J. S. Human Vam6p promotes lysosome clustering and fusion in vivo. J. Cell Biol. 154, 109-122 (2001).

24. Chirivino, D. et al. The ERM proteins interact with the HOPS complex to regulate the maturation of endosomes. Mol. Biol. Cell. 22, 375-385 (2011).

25. Wang, T., Wong, K. \& Hong, W. A unique region of RILP distinguishes it from its related proteins in its regulation of lysosomal morphology and interaction with Rab7 and Rab34. Mol. Biol. Cell 15, 815-826 (2004)

26. Chen, L., Hu, J., Yun, Y. \& Wang, T. Rab36 regulates the spatial distribution of late endosomes and lysosomes through a similar mechanism to Rab34. Mol. Membr. Biol. 27, 24-31 (2010).

27. Wu, M., Wang, T., Loh, E., Hong, W. \& Song, H. Structural basis for recruitment of RILP by small GTPase Rab7. EMBO J. 24, 1491-501 (2005).

28. Johansson, M. et al. Activation of endosomal dynein motors by stepwise assembly of Rab7-RILP-p150Glued, ORP1L, and the receptor betall spectrin. J. Cell Biol. 176, 459-471 (2007).

29. Brett, C. L. et al. Efficient termination of vacuolar Rab GTPase signaling requires coordinated action by a GAP and a protein kinase. J. Cell Biol. 182, 1141-1151 (2008).

30. Cabrera, M. et al. Vps41 phosphorylation and the Rab Ypt7 control the targeting of the HOPS complex to endosome-vacuole fusion sites. Mol. Biol. Cell 20, 1937-1948 (2009).

31. Sakane, A., Hatakeyama, S. \& Sasaki, T. Involvement of Rabring7 in EGF receptor degradation as an E3 ligase. Biochem. Biophys. Res. Commun. 357, 1058-1064 (2007)

32. Jager, S. et al. Role for Rab7 in maturation of late autophagic vacuoles. J. Cell Sci. 117, 4837-4848 (2004)

33. Jordens, I. et al. Rab7 and Rab27a control two motor protein activities involved in melanosomal transport. Pigment Cell Res. 19, 412-423 (2006).

34. Zhang, M., Chen, L., Wang, S. C. \& Wang, T. Rab7: roles in membrane trafficking and disease. Biosci. Rep. 29, 193-209 (2009).

35. LaGrassa, T. J. \& Ungermann, C. The vacuolar kinase Yck3 maintains organelle fragmentation by regulating the HOPS tethering complex. J. Cell Biol. 168 401-414 (2005).
36. Baker, R. W., Jeffrey, P. D. \& Hughson, F. M. Crystal Structures of the Sec1/ Munc18 (SM) Protein Vps33, Alone and Bound to the Homotypic Fusion and Vacuolar Protein Sorting (HOPS) Subunit Vps16. PLoS One. 26, e67409 (2013).

37. Bröcker, C. et al. Molecular architecture of the multisubunit homotypic fusion and vacuole protein sorting (HOPS) tethering complex. Proc Natl Acad Sci U S A. 7, 1991-1996 (2012)

38. Progida, C. et al. RILP is required for the proper morphology and function of late endosomes. J. Cell Sci. 120, 3729-3737 (2007).

39. Kinchen, J. M. \& Ravichandran, K. S. Identification of two evolutionarily conserved genes regulating processing of engulfed apoptotic cells. Nature 464, 778-782 (2010).

40. Rink, J., Ghigo, E., Kalaidzidis, Y. \& Zerial, M. Rab conversion as a mechanism of progression from early to late endosomes. Cell 122, 735-749 (2005).

41. Via, L. E. et al. Arrest of mycobacterial phagosome maturation is caused by a block in vesicle fusion between stages controlled by rab5 and rab7. J. Biol. Chem. 272, 13326-13331 (1997).

42. Peplowska, K., Markgraf, D. F., Ostrowicz, C. W., Bange, G. \& Ungermann, C. The CORVET tethering complex interacts with the yeast Rab5 homolog Vps21 and is involved in endo-lysosomal biogenesis. Dev. Cell 12, 739-750 (2007)

43. Markgraf, D. F. et al. The CORVET subunit Vps8 cooperates with the Rab5 homolog Vps21 to induce clustering of late endosomal compartments. Mol. Biol. Cell 20, 5276-5289 (2009).

44. Wang, T. \& Hong, W. RILP interacts with VPS22 and VPS36 of ESCRT-II and regulates their membrane recruitment. Biochem. Biophys. Res. Commun. 350, 413-423 (2006)

45. Wang, T. \& Hong, W. Interorganellar regulation of lysosome positioning by the Golgi apparatus through Rab34 interaction with Rab-interacting lysosomal protein. Mol. Biol. Cell 13, 4317-4332 (2002).

46. Wang, T. et al. A role of Rab7 in stabilizing EGFR-Her2 and in sustaining Akt survival signal. J. Cell. Physiol. 227, 2788-2797 (2012).

\section{Acknowledgments}

This work was supported by National Natural Science Foundation of China (No.30971442, No.31071176 and No.31371353), and China-Singapore joint research program (No.2013DFG32730).

\section{Author contributions}

T.W. and W.H. designed the experiments and wrote the main manuscript; T.Y. and X.L. prepared the Figure 1-3, Figure 5, Figure s1 and Figure s2, Figure s6; S.W. and Z.W. prepared the Figure 4, Figure $s 3$ and Figure s4; L.S. and X.X. prepared the Figure 6, Figure 7 and Figure s5; Y.Y. and Y.Z. Contributed reagents/materials/analysis tools; C.A. provided the myc-tagged HOPS subunits VPS11, VPS16, VPS18, VPS33 and VPS41. All author reviewed the manuscript.

\section{Additional information}

Supplementary information accompanies this paper at http://www.nature.com/ scientificreports

Competing financial interests: The authors declare no competing financial interests.

How to cite this article: Lin, X. et al. RILP interacts with HOPS complex via VPS41 subunit to regulate endocytic trafficking. Sci. Rep. 4, 7282; DOI:10.1038/srep07282 (2014).

This work is licensed under a Creative Commons Attribution-NonCommercialShareAlike 4.0 International License. The images or other third party material in this article are included in the article's Creative Commons license, unless indicated otherwise in the credit line; if the material is not included under the Creative Commons license, users will need to obtain permission from the license holder in order to reproduce the material. To view a copy of this license, visit http:// creativecommons.org/licenses/by-nc-sa/4.0/ 


\title{
SCIENTIFIC REP

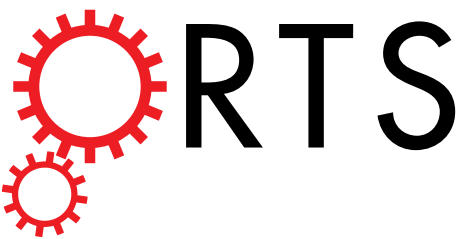 \\ Corrigendum: RILP interacts with HOPS complex via VPS41 subunit to regulate endocytic trafficking
}

\author{
Xiaosi Lin, Ting Yang, Shicong Wang, Zhen Wang, Ye Yun, Lixiang Sun, Yunhe Zhou, \\ Xiaohui Xu, Chihiro Akazawa, Wanjin Hong \& Tuanlao Wang
}

Scientific Reports 4:7282; doi: 10.1038/srep07282; published online 2 December 2014; updated 15 July 2015

We did not cite a related study which demonstrated that RILP facilitates membrane recruitment of tethering HOPS complex and p150glued subunit of dynein complex, which is regulated by cholesterol sensor ORP1L (Reference 1). In that investigation, RILP interacts with HOPS complex and recruits subunits of HOPS complex to late endosomal membrane, which is consistent with our results. However, the data from van der Kant et al. suggested that multiple sites of RILP may bind to various subunits of HOPS complex, while our results strongly suggest that RILP preferentially interacts with accessory subunit VPS41, this discrepancy may be due to different approaches for testing the interaction (surface plasmon resonance (SPR) by van der Kant et al. vs GST-pulldown by us). We further characterized the region in VPS41 that is responsible for the interaction with RILP, and also functionally showed that the interaction of RILP with VPS41 is responsible for the endocytic trafficking of EGFR. We also revealed that the interaction of RILP with HOPS complex is independent of Rab7. We propose that RILP plays a role in facilitating the formation and stabilizing of Rab7-RILP-HOPS supercomplex on late endosomal membranes. Together with the results by Kant $e t$ al, RILP interaction with HOPS complex is a novel mechanism for regulating endosomal trafficking.

\section{References}

1. van der Kant, R. et al. Late endosomal transport and tethering are coupled processes controlled by RILP and the cholesterol sensor ORP1L. J. Cell Sci. 126, 3462-3474 (2013) 